\title{
Ansteckung in der Wertschöpfungskette
}

\author{
Johannes Matt
}

\section{Relevanz}

Ob Sturm, Feuer, Streik, Managementversagen oder Handelskonflikte, die Risiken der Unternehmen sind zahlreich. Gefahr droht auch, wenn es den Lieferanten und Kunden schlecht geht. Fällt ein schwer ersetzbarer Lieferant aus, oder geht einem wichtigen Kunden das Geld aus, dann herrscht Krise. Je stärker die Arbeitsteilung und Spezialisierung in der Produktion ist, desto mehr sind die Unternehmen in einem Netzwerk von Lieferbeziehungen eng verflochten und voneinander abhängig. Umso verhängnisvoller kann sich ein Unterbruch in der Produktionskette auswirken. Die Ansteckung in der Wertschöpfungskette kann ganze Branchen erfassen und Konjunkturabschwünge verschärfen. Um die Krisenrobustheit zu stärken, können die Unternehmen z. B. mit Lagerhaltung, Diversifizierung der Lieferanten und ausreichenden Kapitalreserven vorsorgen.

\section{Quelle}

Barrot, J.-N. und Sauvagnat, J. (2016), Input Specificity and the Propagation of Idiosyncratic Shocks in Production Networks, Quarterly Journal of Economics $131,1543-1592$.

Unternehmen sind durch eine Vielzahl von Lieferbeziehungen eng miteinander verflochten. Dadurch kann sich die finanzielle Schieflage eines Lieferanten schnell zu einem Geschäftsrisiko für seine Kunden und sogar für eine ganze

J. Matt $(\bowtie)$

Universität St.Gallen, St.Gallen, Schweiz

E-Mail: J.I.Matt@1se.ac.uk 
Branche entwickeln. Die Schocks und ihre Auswirkungen können so stark sein, dass sie ganze Industriesektoren beinträchtigen und damit die Konjunktureinbrüche verschärfen. Die ökonomische Forschung hat sich bisher vor allem mit der Verflechtung zwischen speziellen Branchen und ihrem Einfluss auf die Gesamtwirtschaft beschäftigt. Wie die komplexen Wertschöpfungsketten von den Verflechtungen zwischen einzelnen Firmen abhängen, ist noch kaum bekannt. Das analysieren die Ökonomen Jean-Noël Barrot (MIT) und Julien Sauvagnat (Bocconi). Sie untersuchen, wie sich Schocks wie z. B. Produktionsausfälle bei einem einzelnen Unternehmen auf andere Unternehmen in der Wertschöpfungskette, die nicht direkt davon betroffen sind, ausweiten.

Jedes Unternehmen ist Teil eines mehr oder weniger komplexen Netzwerkes von Lieferanten, Sublieferanten und Kunden. Unternehmen beliefern sich gegenseitig und vertreiben ihre Produkte auf einem gemeinsamen Markt. Wenn eines dieser Unternehmen seine Produktion unterbrechen muss oder es aus anderen Gründen zu Lieferengpässen kommt, müssen seine Kunden darauf reagieren. Diese Reaktion wird von zwei Faktoren bestimmt.

Einerseits können Unternehmen einen Großteil solcher Schocks durch eigene Vorkehrungen und Anpassungen in ihrer Produktion ausgleichen. Die Aufteilung der Einkäufe auf verschiedene Lieferanten sowie die Lagerhaltung sind Beispiele dafür. Selbst bei einer starken Unterbrechung und bei Lieferengpässen und Preissteigerungen sind viele Unternehmen flexibel genug, ihre Produktion anzupassen oder auf andere Lieferanten auszuweichen. Andererseits können langfristige Lieferverträge, die Exklusivität eines Lieferanten oder Patente solche Anpassungen erschweren. In diesem Fall breiten sich Schocks in einem speziellen Unternehmen schneller und stärker im Produktionsnetzwerk aus und können sich gegenseitig aufschaukeln. Gerät ein Unternehmen in Schwierigkeiten, dann bekommen das seine Kunden und Lieferanten stark zu spüren. Je größer das betroffene Unternehmen, je rigider die Produktion, oder je verzweigter das Netzwerk zu anderen Firmen ist, desto stärker breitet sich ein Schock aus, und desto größer fallen die Auswirkungen auf die Gesamtwirtschaft aus.

Ein Beispiel für ein Firmennetzwerk ist in Abb. 1 zu sehen. Es zeigt die Entwicklung von Beziehungen zwischen Lieferanten und Kunden im Zeitraum von 1995 bis 2000. Verbindungen, die zu Beginn und am Ende der Periode bestanden, sind dunkel hinterlegt. Alle anderen Beziehungen sind entweder vor dem Jahr 2000 abgebrochen oder erst nach 1995 aufgenommen worden. Die Distanz zwischen zwei Punkten zeigt die geographische Entfernung der Unternehmen zueinander, die Dicke die Stärke der Beziehung.

Die Abbildung illustriert die Komplexität eines Lieferanten- und Kundennetzwerks und weist auf die Folgen eines Schocks hin. Dabei haben nicht alle Unternehmen die gleiche Bedeutung in der Wertschöpfungskette. Ein Aus- 


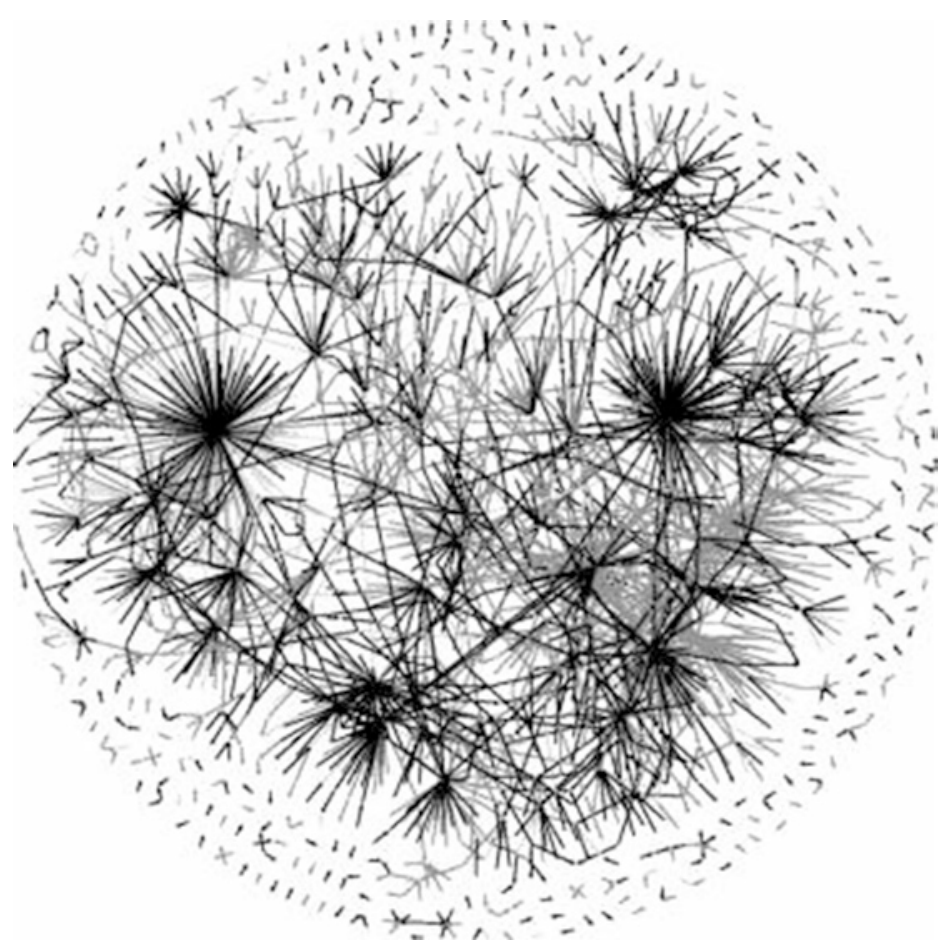

Abb. 1 Exemplarisches Firmennetzwerk aus Kunden und Lieferanten, 1995-2000. (Quelle: Barrot und Sauvagnat 2016)

fall der Produktion an einem der Knotenpunkte, also bei einem stark vernetzten Leitbetrieb, beeinträchtigt eine große Zahl von Unternehmen im Netzwerk. In der Abbildung lässt sich zudem erkennen, dass Verbindungen zu geographisch nahegelegenen Unternehmen bevorzugt werden. Ein Produktionsschock wirkt sich daher typischerweise vor allem auf die Kunden und Zulieferer in der nahen Umgebung schwerwiegend aus.

Die Ökonomen haben es nicht immer einfach, solche Wirkungsketten festzumachen und empirisch eindeutig zu identifizieren. Eine Vielzahl von Einflussfaktoren macht es oft nahezu unmöglich, den direkten Effekt bei einem einzelnen Unternehmen zu isolieren. Ereignisse, die ein Unternehmen ganz allein betreffen, sind selten und schwierig auszumachen. Barrot und Sauvagnat wenden daher einen speziellen Trick an. Sie untersuchen für die USA zwischen 1978 und 2013, wie sich lokal begrenzte Naturkatastrophen wie z. B. Feuer, Sturm, Überflutung 
oder starker Schneefall auf die betroffenen Unternehmen mit ihren vor- und nachgelagerten Partnern auswirken. Dann messen sie den Unterschied zum Geschäftsgang bei vergleichbaren Firmen, die nicht direkt unter der Katastrophe leiden, aber durch ihr Netzwerk mit den betroffenen Unternehmen verflochten sind. Je stärker der Schock und je bedeutsamer die Rolle eines Unternehmens im Netzwerk ist, desto stärker sollten die Auswirkungen eines solchen Schocks für andere Unternehmen zu spüren sein.

Bei einem lokal begrenzten Schock wie z.B. einer Naturkatastrophe geht das Umsatzwachstum des betroffenen Unternehmens um durchschnittlich 5 Prozentpunkte und bei den Kunden um 2-3 Prozentpunkte zurück.

Zuerst schätzen die Forscher, dass das Umsatzwachstum eines Unternehmens, das von einer Naturkatastrophe getroffen wird, um durchschnittlich 5 Prozentpunkte zurückgeht. Dies sollte einen direkten Einfluss auf den Umsatz der Kunden haben. Tatsächlich schätzen Barrot und Sauvagnat, dass ein solcher Schock den Umsatz der Kunden um 2 bis 3 Prozentpunkte reduziert. Dies entspricht einem Rückgang von $25 \%$ im Vergleich zur durchschnittlichen Wachstumsrate über drei Quartale.

Zusätzlich zu den Auswirkungen auf das Umsatzwachstum untersuchen die Wissenschaftler die Entwicklung der Eigenkapitalwerte von börsennotierten Unternehmen. Abb. 2 verfolgt die Entwicklung der Börsenwerte gemessen am

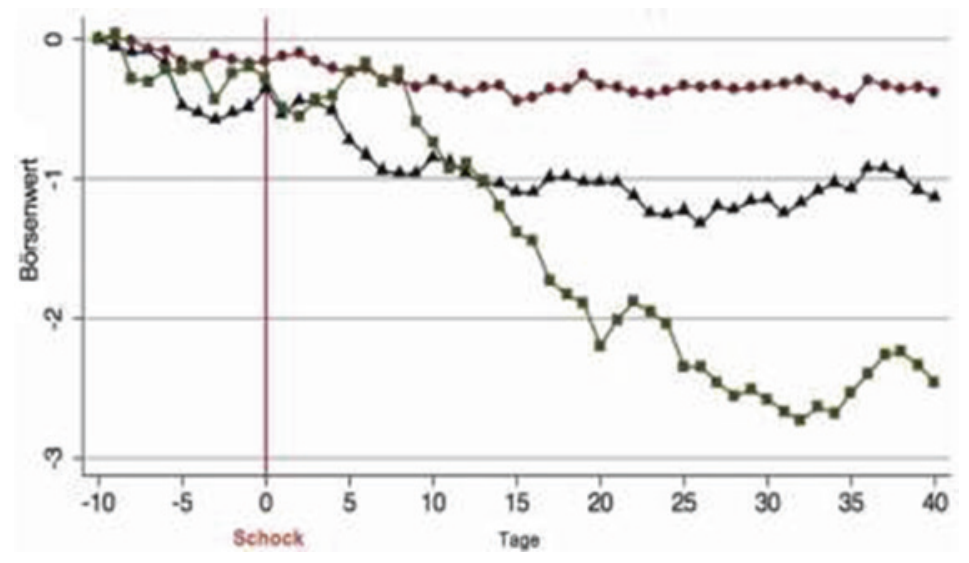

Abb. 2: Unternehmenswert eines betroffenen Lieferanten und seiner Kunden nach einem Schock. (Quelle: Barrot und Sauvagnat 2016, S. 1578) 
Aktienkurs eines direkt betroffenen Lieferanten (untere, grüne Linie) und seiner typischen Kunden (mittlere, blaue Linie) sowie eines nicht-betroffenen vergleichbaren Mittbewerbers (obere, rote Linie) und verdeutlicht, wie sich die Börsenwerte nach einem Schock auseinanderentwickeln.

Die vertikale Linie bezeichnet den Zeitpunkt der Naturkatastrophe und die vertikale Achse den Verlust im Aktienwert in Prozent. Im ersten Monat nach dem Schock reduziert sich der Börsenwert des betroffenen Lieferanten um durchschnittlich $3 \%$, während sich jener des Kunden um bis zu $1 \%$ verringert, also um ein Drittel davon.

Nach einem Produktionsstopp fällt der Börsenwert eines betroffenen Unternehmens um durchschnittlich 3 Prozent. Das führt in einem Firmennetzwerk zu einem Wertverlust bei den Kunden von bis zu 1 Prozent des Börsenwerts.

Zudem breitet sich ein Schock auch horizontal auf andere Unternehmen aus, die zwar selbst nicht direkt betroffen sind, aber Kunden beliefern, die auch bei den direkt betroffenen Lieferanten einkaufen. Auch solche anderen, nicht direkt betroffenen Firmen stecken sich an, indem sie auf dem Umweg über ihre Kunden ein um bis $\mathrm{zu}$ vier Prozentpunkte geringeres Umsatzwachstum hinnehmen müssen. Die Krise eines Unternehmens breitet sich also nicht nur vertikal entlang einer Wertschöpfungskette aus, sondern auch horizontal zwischen Lieferanten auf derselben Verarbeitungsstufe. So kann sich ein ganzes Netzwerk oder eine ganze Branche anstecken.

Die Schätzungen von Barrot und Sauvagnat beschränken sich zwar nur auf die Effekte von Naturkatastrophen. Mit ihrem Ansatz können sie aber Schocks identifizieren, die zunächst nur auf einzelne Unternehmen beschränkt sind, und können so die Fortpflanzungseffekte separat herausfiltern. Wie die Wissenschaftler betonen, lassen sich ihre Ergebnisse mit großer Wahrscheinlichkeit auch auf andere Unterbrechungen der Wertschöpfungskette z. B. infolge von Streiks, Management-Wechsel oder Handelskonflikte verallgemeinern. Es kommt nicht auf die Ursache des Produktionsausfalls an, sondern auf die Bedeutung der Güter und Dienstleistungen, welche ein Kunde von seinen Lieferanten bezieht. Je schwieriger es für einen Kunden ist, einen ausfallenden Lieferanten zu ersetzen, desto stärker wird er von einem Unterbruch in der vorausgehenden Verarbeitungsstufe betroffen sein. Dies betrifft insbesondere Branchen, die einer starken Regulierung unterliegen, und in welchen Patente von wenigen Anbietern gehalten werden. Dies kann die Ansteckungseffekte von unternehmensspezifischen Schocks signifikant erhöhen. 
Open Access Dieses Kapitel wird unter der Creative Commons Namensnennung 4.0 International Lizenz (http://creativecommons.org/licenses/by/4.0/deed.de) veröffentlicht, welche die Nutzung, Vervielfältigung, Bearbeitung, Verbreitung und Wiedergabe in jeglichem Medium und Format erlaubt, sofern Sie den/die ursprünglichen Autor(en) und die Quelle ordnungsgemäß nennen, einen Link zur Creative Commons Lizenz beifügen und angeben, ob Änderungen vorgenommen wurden.

Die in diesem Kapitel enthaltenen Bilder und sonstiges Drittmaterial unterliegen ebenfalls der genannten Creative Commons Lizenz, sofern sich aus der Abbildungslegende nichts anderes ergibt. Sofern das betreffende Material nicht unter der genannten Creative Commons Lizenz steht und die betreffende Handlung nicht nach gesetzlichen Vorschriften erlaubt ist, ist für die oben aufgeführten Weiterverwendungen des Materials die Einwilligung des jeweiligen Rechteinhabers einzuholen.

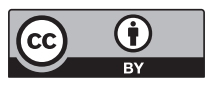

